\title{
Late presentation of breast carcinoma at North Central Province of Sri Lanka.
}

\author{
WAK Weerawardena, PDJ Edirisooriya, IJ Piyadasa, TDB Illangasingha, GAL \\ Niroshana, SM Rathnayaka, WTDUPT Subaweera.
}

Surgical Unit - Teaching Hospital Anuradhapura

\begin{abstract}
Introduction

Breast carcinoma is the main cancer among female. Delay in presentation causes high morbidity and mortality world wide. Our aim was to analyze female breast carcinoma at North Central Province in relation to delay in seeking $\mathrm{t} r$ e a $\mathrm{t} m$ e $\mathrm{n} \mathrm{t}$
\end{abstract}

\section{Objective}

Analyze presentation of patients with breast carcinoma to surgical wards in Anuradhapura and Polonnaruwa Hospital.

\section{Materials and methods}

We analyzed all the patients who underwent mastectomy for carcinoma in surgical unit B at General Hospital Polonnaruwa (from July 2007 to September 2009) and at Teaching Hospital Anuradhapura (from September 2009 to June 2012).

\section{Results}

We studied 44 patients. Duration of delay in presentations was with a range of 1 month to 3 years (mean 8 months). Only 11(25\%) presented within 3 months of first detecting symptoms. Seventeen (45\%) cases were detected accidentally, pain has given the attention in $18(47 \%)$ cases while only $3(8 \%)$ cases were detected during self breast examination(SBE) out of 38 cases.

\section{Conclusion}

We need to broaden the knowledge of people in the community about symptoms of carcinoma including non lump symptoms and consequences. People should be encouraged to perform SBE and present early.
It is necessary to overcome the barriers of disclosing the symptoms to another and barriers to approach the health care institutions considering socioeconomic status of patients.

\section{Introduction}

Breast carcinoma is the commonest cancer in women worldwide. This cancer is the main cause of death from cancer among women with approximately 1.3 million new cases and an estimated 458000 deaths reported in 2008 in the world. In the United States a woman has a 1 in 8 chance of having invasive breast carcinoma during her lifetime. The breast carcinoma risk increases with age. Tumour stage is the main determinant of breast carcinoma outcome of women. Among female nonmetastatic breast carcinoma, there is a strong correlation between tumour size and the extent of axillary spread. The risk of distant metastasis is most closely correlated with the number of axillary lymph nodes involvement, followed by tumour size. This means that the ideal screening regimen for breast cancer would be one that could detect a tumour before it was large enough to be palpable. Since 1990, mortality from breast carcinoma in the United States and other industrialized countries has been decreasing at the rate of approximately $2.2 \%$ per year. In the United States, this decline has been attributed both to advances in adjuvant therapy and to increasing use of screening mammography, in approximately equal measure ${ }^{1}$. We have analyzed the data of patients presented with breast carcinoma to detect the time of presentation for intervention. 


\section{Materials and methods.}

We have analyzed 44 patients who underwent mastectomy for breast carcinoma from July 2007 to September 2009 at surgical unit B at General Hospital Polonnaruwa and from September 2009 to June 2012 at Teaching Hospital Anuradhapura. The age, sex, site and size of the breast lump at presentation, detection method, delay in presentation and outcome of the patients were analyzed. During the search we have analyzed about the possible aetiological factors for the breast carcinoma such as use of oral contraceptive pills, Depo Provera, the age at menarche, menopausal age, age at first child birth and breast feeding. All the patients with suspected breast lumps were investigated with triple assessment (clinical examination, ultrasound examination and fine needle aspiration cytology (FNAC). We did not perform mammogram in any of those patients. If FNAC report is inconclusive we proceeded to perform incisional biopsy. Although we offered breast reconstruction procedures to the patients, they were not willing for such surgeries. If the cancer is at advanced stage we offered neoadjuvent chemotherapy. We performed Patey mastectomy with level 3 axillary clearance in those studied patients. All the patients were discharged at $5^{\text {th }}$ day with the removal of draitube.

\section{Results}

There were 44 patients (19 patients at GH Polonnaruwa and 25 patients at $\mathrm{TH}$ Anuradhapura). All the patients were females with age distribution of 31-74 years (mean 51.4). Maximum lengths of the lesions were with a range from $2 \mathrm{~cm}$ to $8 \mathrm{~cm}$ (mean $4.0 \mathrm{~cm}$ ). Twenty six were right side and 18 were left sided. Duration of delay in presentations was with a range of 1 month to 3 years (mean 8 months). Only 11 (25\%) presented within 3 months of first detecting a lump.
Late presentation of breast carcinoma at North Central Province of Sri Lanka. Age distribution of 25 patients from Anuradhapura.

\begin{tabular}{|c|c|}
\hline Age group & Number of patients \\
\hline $31-40$ & 05 \\
\hline $41-50$ & 10 \\
\hline $51-60$ & 06 \\
\hline $61-70$ & 01 \\
\hline $71-80$ & 03 \\
\hline
\end{tabular}

Analyzing the possible aetiological factors showed only 13 (31\%) used hormonal contraceptive method. They attained menarche at 12-16 years of age. Mean menopausal age was 46.8 years (range $37-54$ years). Mean age at first child birth was 23.5 years (range 13-39) of 19 cases. Thirty three of 36 (91\%) have breast fed and $24(56 \%)$ of 43 patients have more than 2 children

Seventeen (45\%) patients detected lump accidentally. Pain has given the attention in $18(47 \%)$ cases while only $3(8 \%)$ cases were detected during self breast examination out of 38 cases. One patient has died shortly with metastasis. All the other patients are following oncology clinics

\section{Discussion}

Delayed patient presentation refers to a prolonged interval between discovery of initial symptoms to presentation to a provider and is typically defined as greater than 12 weeks. Delayed presentation of breast cancer $(\geq 3$ months) is associated with poorer survival at all ages $^{2,3}$ The intervention builds on evidence about risk factors for delayed presentation of breast cancer. The ultimate aim of the intervention is to reduce the proportion of older women with breast cancer who delay their presentation, and thereby save lives. 
Self breast examination (SBE) is helpful for early detection of breast carcinoma. In one study done in Iran, significant proportion of people studied performed SBE .Proportion of those patients did not perform self breast examination because of lack of knowledge about the technique of examination. Some patients did not perform SBE because of fear of becoming positive for carcinoma or did not care about them. A large proportion of patients who perform SBE do it incorrectly. Those who performed SBE learned it from medical personals, their relatives, television, radio, books, journals and pamphlets. In our study sample only $8 \%$ of patients did $\mathrm{SBE}$.

Community disease preventive teams should teach of Self Breast Examination [SBE] to patients, Clinical Breast Examination [CBE] to health workers. All efforts should be made to break the vicious cycle of late presentation, poor treatment outcome and reluctance of patients to present to health facilities because of poor outcome. Significant delays in presentation still abound with only $12 \%$ presenting within 1 month of noticing symptoms in one study in Nigeria. This late presentation is universal among patients in the third world including Asian and Arab countries. The reasons for delayed presentation included long distances to hospital, lack of awareness, fear of the consequences, strong belief in traditional medicine, religious charlatans, poverty, poor education, fear, denial, and negligence by patients or their family members and general practitioners." This knowledge can be used to launch a program to motivate the patients to present early in North Central Province in Sri Lanka.

In one study Breast cancer risk was found to be increased in women with age $(\geq 50)$, induced abortion, age at first birth ( $\geq 35$ years), body mass index (BMI $\geq 25$ ), and a positive family history. However, decreased breast cancer risk was associated with the duration of education $(\geq 13$ years), presence of spontaneous abortion, breast feeding $^{10}$
These factors need to be considered for targe ting a population in planning of education pro grams of breast carcinoma. However the asso ciation of high breast carcinoma risk with lower duration of education may be a barrier for pro per communication with health workers.

Women need further information about the different types of breast cancer symptoms to assist symptom recognition, as well as encouragement to seek medical advice if a symptom is ambiguous. In addition, women may benefit from greater awareness of the benefits of early detection and reassurance about the improvements in quality of breast cancer care ${ }^{11}$. Those non lump symptoms are known factors to delay in presentation. It is necessary to broaden the knowledge of patients about the symptoms of breast carcinoma. ${ }^{11}$ Those patients who present late due to fear of consequences of carcinoma usually gathered the information from their friends and relatives. People should be educated that if detect early, lesser degree of surgeries are available in cancer treatment. If presentation is late extensive surgeries with radio/chemotherapy methods are needed for therapeutic purpose $\mathrm{e}^{11.12}$

Some of the patients do not attend to seek treatment because they failed to prioritize the health ov e r their other activities. So education of breast cancer awareness programs should extent to educate the consequence of not attending for treatment

Symptoms that could be a warning sign of breast cancer included a painless lump, swelling, breast skin changes, pain, discharge from the nipples, nipple retraction. Fever, pruritus, cold, weight loss and presence of a wound have been recognized as presentation symptoms of women. In a study in Nigeria only few people knew that BSE should be performed 2-3 days after menstruation monthly and less number knew that women who have reached menopause were expected to choose a specific day of the month to perform BSE. 
One review article shows poverty to be the most common and strongest barrier for early presentation. Traveling away from home, family and work responsibilities, and high cost of diagnosis and treatment constitute particularly burdensome problems facing the poor. Future interventions should primarily attempt to enhance access for affordable healthcare close to their home (3).

\section{Conclusion}

It is necessary to design programs to increase women's knowledge about breast symptoms and risk, to promote disclosure of symptoms to someone, to reduce the barriers to approach therapy. There are many barriers to overcome to achieve these targets. However all these are feasible with the available facilities in North Central Province.

\section{References}

\section{1 ) Ellen Warner. Cancer Screening. N Engl J} Med 2011; 365:1025-1032.

2) AJ Ramirez, AM Westcombe, CC Burgess, S Sutton, P Littlejohns, MA Richards. "Factors predicting delayed presentation of symptomatic breast cancer: a systematic review," The Lancet 1999;353(9159):1127-1131.

3) Ketan Sharma, Ainhoa Costas, Lawrence N. Shulman, John G. Meara. Review Article. A

Systematic Review of Barriers to Breast Cancer Care in Developing Countries Resulting in Delayed Patient Presentation. Journal of Oncology Volume $2012 ; 2012: 8$ pages.

4) A Ramirez. Promoting early breast cancer presentation in women after their final routine breast screening mammogram Breast Cancer Research 2006;8(Suppl 1):15.

5) A Simi, M Yadollahie, F Habibzadeh. Knowledge and attitudes of breast self examination in a group of women in Shiraz, southern Iran . Postgrad Med J 2009;85:283287.
6) G Ertem, A Kocer. Breast self-examination among nurses and midwives in Odemis health district in Turkey. Indian journal of cancer 2009 ; $46(3$ ): 208-213.

7) TT Fancher, J A Palesty, JJ Paszkowiak, RP Kiran, AD Malkan, SJ Dudrick . Can Breast Self-Examination Continue to Be Touted Justifiably as an Optional Practice? International Journal of Surgical Oncology 2011;2011:1-5.

8) Stanley NC Anyanwu . Temporal trends in breast cancer presentation in the third world. Journal of Experimental \& Clinical Cancer Research 2008;27:17.

9) Talpur AA, Surahio AR, Ansari A, Ghumro AA Late presentation of breast cancer: a dilemma. JPMA, The Journal of the Pakistan Medical Association 2011;61(7):662-666.

10) Vahit Ozmen, Beyza Ozcinar, Hasan Karanlik, Neslihan Cabioglu, Mustafa Tukenmez, Rian Disci, Tolga Ozmen, Abdullah Igci, Mahmut Muslumanoglu, Mustafa Kecer, Atilla Soran. Breast cancer risk factors in Turkish women - a University Hospital based nested case control study World Journal of Surgical Oncology 2009;7:37.

11) C Burgess, MS Hunter, AJ Ramirez. A qualitative study of delay among women reporting symptoms of breast cancer. Br J Gen Pract 2001 December; 51(473): 967-971.

12) Al-Kahiry W, Omer HH, Saeed NM, Hamid GA. Late presentation of breast cancer in aden, yemen. Gulf J Oncolog 2011 Jan; 1(9):7-11.

13 ) Abimbola Oluwatosin, Oladimeji Oladepo. Knowledge of breast cancer and its early detection measures among rural women in Akinyele Local Government Area, Ibadan, Nigeria. BMC Cancer 2006; 6:271.

14) Bachok Norsa'adah, Krishna G Rampal, Mohd A Rahmah, Nyi N Naing, Biswa M Biswal. Diagnosis delay of breast cancer and its associated factors in Malaysian women . BMC Cancer 2011; 11:141. 